\title{
The Potential Impact of Bystander Effects on Radiation Risks in a Mars Mission
}

\author{
D. J. Brenner ${ }^{1}$ and C. D. Elliston \\ Center for Radiological Research, Columbia University, New York, New York 10032
}

\begin{abstract}
Brenner, D. J. and Elliston, C. D. The Potential Impact of Bystander Effects on Radiation Risks in a Mars Mission. Radiat. Res. 156, 612-617 (2001).
\end{abstract}

Densely ionizing (high-LET) galactic cosmic rays (GCR) contribute a significant component of the radiation risk in free space. Over a period of a few months-sufficient for the early stages of radiation carcinogenesis to occur-a significant proportion of cell nuclei will not be traversed. There is convincing evidence, at least in vitro, that irradiated cells can send out signals that can result in damage to nearby unirradiated cells. This observation can hold even when the unirradiated cells have been exposed to low doses of low-LET radiation. We discuss here a quantitative model based on the $\mathcal{B} \mathbf{a} \mathcal{D}$ formalism, an approach that incorporates radiobiological damage both from a bystander response to signals emitted by irradiated cells, and also from direct traversal of high-LET radiations through cell nuclei. The model produces results that are consistent with those of a series of studies of the bystander phenomenon using a high-LET microbeam, with the end point of in vitro oncogenic transformation. According to this picture, for exposure to high-LET particles such as galactic cosmic rays other than protons, the bystander effect is significant primarily at low fluences, i.e., exposures where there are significant numbers of untraversed cells. If the mechanisms postulated here were applicable in vivo, using a linear extrapolation of risks derived from studies using intermediate doses of high-LET radiation (where the contribution of the bystander effect may be negligible) to estimate risks at very low doses (where the bystander effect may be dominant) could underestimate the true risk from low doses of high-LET radiation. It would be highly premature simply to abandon current risk projections for high-LET, low-dose radiation; however, these considerations would suggest caution in applying results derived from experiments using high-LET radiation at fluences above $\sim 1$ particle per nucleus to risk estimation for a Mars mission. $\odot 2001$ by Radiation Research Society

\section{INTRODUCTION}

A decision to proceed with a manned Mars mission will depend on many factors, one of the most critical being the

${ }^{1}$ Author to whom correspondence should be addressed at Center for Radiological Research, Columbia University, 630 West 168th Street, New York, NY 10032; e-mail: djb3@ columbia.edu. risk of radiation-induced stochastic biological effects, particularly cancer. As opposed to radiation exposure in Earth orbit, and with the possible exception of rare large solar particle events, the majority of radiation risk in free space will come from densely ionizing (high-LET) galactic cosmic rays (GCR) (1).

Spatially, as illustrated in Fig. 1, high-LET radiations deposit their energy, and thus produce damage, in an extremely inhomogeneous manner-far less homogeneous than low-LET radiation. Thus, at low and even intermediate doses of high-LET radiation, significant numbers of cells will not be traversed by a high-LET radiation track. ${ }^{2}$ This is illustrated quantitatively in Figs. 2 and 3 (derived from data in ref. 1), which show estimates of the proportion of cell nuclei that remain untraversed by a GCR track as a function of time spent in deep space, shielding and solar cycle position. For example, over a 2-year mission, few cell nuclei will remain untraversed by GCR tracks, but over a period of a few months-sufficient perhaps for the initial stages of radiation carcinogenesis to occur-a significant proportion of cell nuclei will not be traversed. This observation does not depend strongly on the amount of shielding present, but does depend somewhat on the solar cycle (Figs. 2 and 3 ).

The potential significance of untraversed cells in a highLET radiation field is that there is convincing evidence, at least in vitro, that irradiated cells can send out signals which can result in damage to nearby unirradiated cells. The evidence is particularly strong in the case of high-LET radiation for a broad variety of end points $(2-16)$. Some recent results suggest that bystander effects can be induced by high-LET radiation even when the bystander cells have been exposed previously to low doses of $\mathrm{X}$ rays (17).

This bystander effect for high-LET radiation is relevant to situations in which some cells are traversed by a highLET radiation track but a significant number of cells are not, at least in an appropriate time frame for development of oncogenic damage. Such scenarios include home-dwell-

${ }^{2}$ In this context, we consider a cell which is traversed only by lowLET radiation, including $\delta$ rays from an HZE ion, to be an untraversed bystander with respect to a high-LET radiation track. Thus, for example, all the cells in Fig. 1 except the central one would be considered to be bystander cells. 


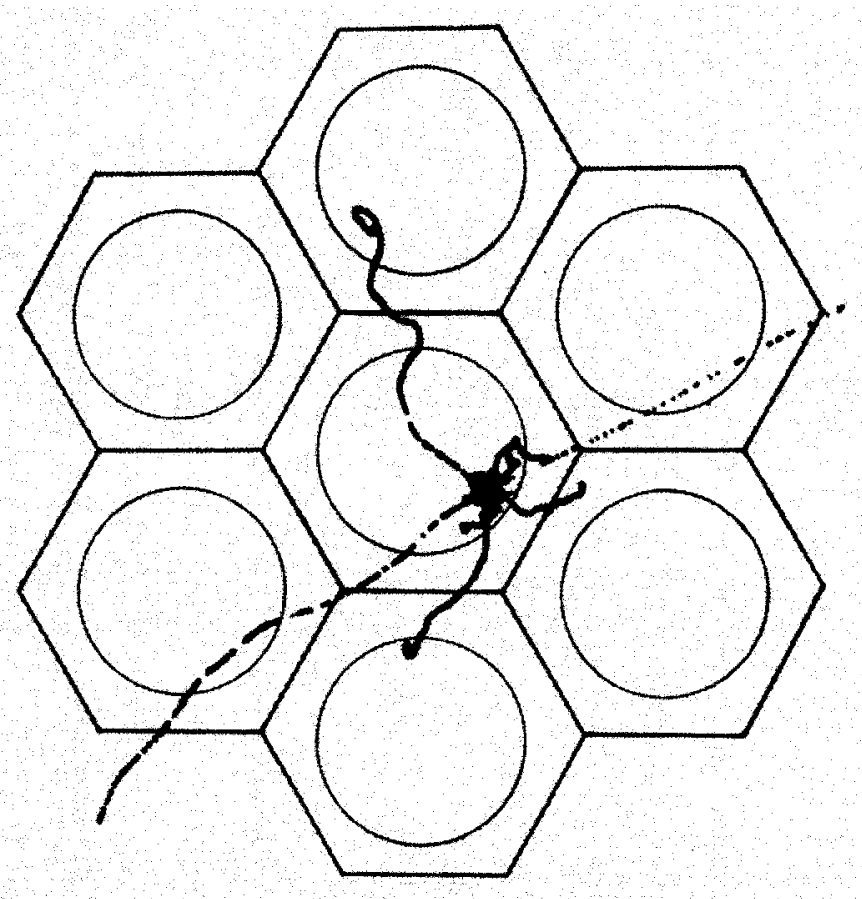

FIG. 1. Slice of an end-on view of a simulation of a $370 \mathrm{MeV} /$ nucleon argon ion traversing a human lens epithelium. Each point represents a single ionization event.

ers exposed to domestic levels of radon and workers exposed occupationally to neutrons. In addition, and of concern here, there is the situation of astronauts in deep space (i.e. outside the Earth's magnetic field), who may receive significant exposures from high-LET galactic cosmic rays.

Currently, as we have no in vivo-based quantitative information about bystander phenomena, we need to rely on insights that we can gain from in vitro studies, though it would be appropriate to maintain a certain skepticism regarding the applicability of in vitro studies of this multicell phenomenon to the situation in vivo.

\section{METHODS}

Studies that have been undertaken at Columbia University to probe the bystander phenomenon have two particularly pertinent features:

1. An in vitro oncogenic transformation assay (using immortalized mouse $\mathrm{C} 3 \mathrm{H}_{10 \mathrm{~T}^{1} / 2}$ cells) was used, a system with potential relevance to the dose-related parts of the process of radiation carcinogenesis in vivo (17; also see Discussion).

2. The use of a single-cell/single-particle microbeam irradiation facility $(19,20)$ has made it possible to define precisely what proportion of cells are traversed by an exactly defined number of $\alpha$ particles, rather than relying on estimates of probabilities.

The goal of these studies was to compare induced oncogenic risks per surviving cell in populations of cells, sparsely plated on dishes (mean nearest-neighbor distance $>50 \mu \mathrm{m}$ ), in which (a) all cell nuclei were hit with precisely defined numbers of high-LET $(95 \mathrm{keV} / \mu \mathrm{m}) \alpha$ particles, and (b) only a small proportion of the cells were hit with the same numbers of $\alpha$ particles, the rest receiving no direct radiation exposure (16). In addition, in earlier studies (21), the risks of oncogenic transformation

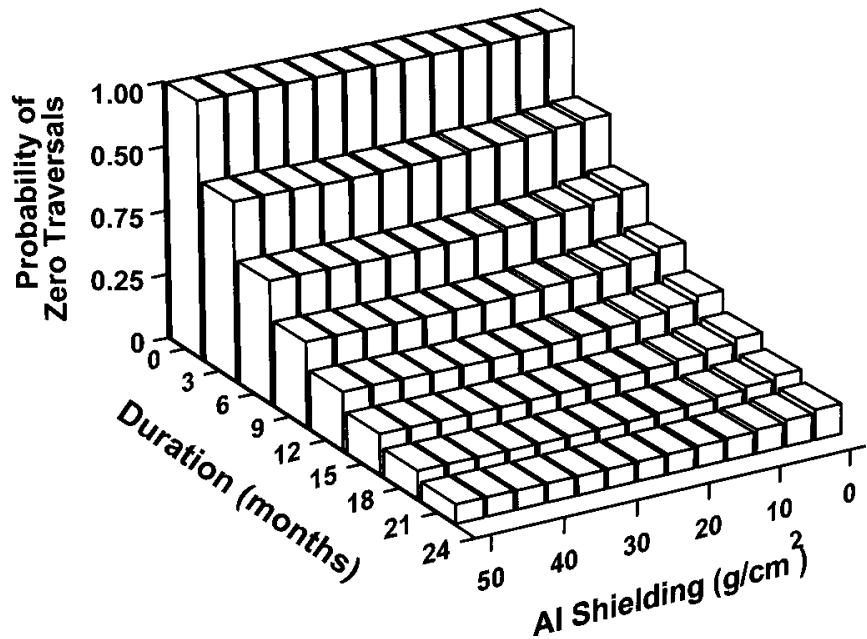

FIG. 2. Estimated probability that a nucleus in a blood-forming organ will not be traversed by the track core of a galactic cosmic ray, as a function of shielding and of duration in free space. The data are for an average solar cycle position. It is of interest to note that, as the amount of shielding is increased, the changes in dose and in LET combine to make the proportion of nonhit cells relatively insensitive to the amount of shielding. Results derived from data in ref. (1).

per surviving cell when all cell nuclei received exactly the same number of $\alpha$ particles were compared with the corresponding risks when cells were exposed to a Poisson-distributed number of $\alpha$ particles - the situation which actually applies for external high-LET radiation exposure.

\section{RESULTS}

\section{In Vitro Oncogenic Transformation Rates}

In the experiments (21) comparing a Poisson-distributed number of $\alpha$-particle traversals with the situation in which each cell nucleus received the same known number of traversals, the risks were essentially the same, except for the one case for $\alpha$ particles, where a Poisson mean of one $\alpha$ particle produced a significantly larger risk than when all cell nuclei were traversed by exactly one $\alpha$ particle.

For exposures to exactly two or more $\alpha$ particles, com-

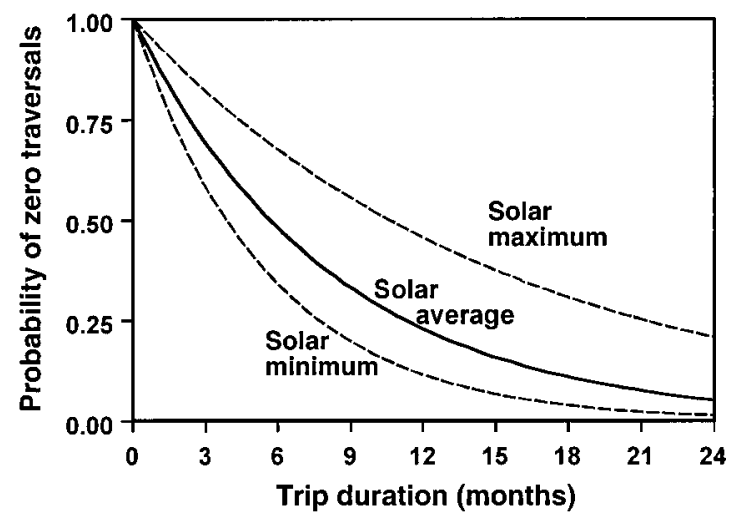

FIG. 3. Estimated probability that a nucleus in a blood-forming organ will not be traversed by the track core of a galactic cosmic ray, as a function of solar cycle position and of duration in free space. Results derived from data in ref. (1), for $20 \mathrm{~g} / \mathrm{cm}^{2}$ of aluminum shielding. 
parisons (16) between microbeam exposure of all cells on a dish compared to exposure of only 1 in 10 cells on a dish showed that the resulting induced oncogenic transformation frequencies were statistically indistinguishable. For the one case for $\alpha$ particles, when $10 \%$ of the population was exposed to exactly one $\alpha$ particle, the induced transformation rate was larger than the situation in which all the cells were exposed to exactly one $\alpha$ particle.

Because of these surprising results, and because of the particular relevance of the results after traversal of cells by one high-LET particle, we subsequently performed two repeats of the part of the experiment in which $10 \%$ of the cells were exposed to exactly one $\alpha$ particle. Unlike the previous experiments, no control experiments were performed for the repeat studies. Based on a standard test for homogeneity (22), we were not able to pool the results of the later experiments with those of the original experiment, although these combined results show an increased transformation frequency of about a factor of two for the case in which $10 \%$ of the cells were exposed to one $\alpha$ particle, compared with the case in which $100 \%$ of the cells were exposed to exactly one $\alpha$ particle. However, no statistical comparisons are possible for the combined results.

\section{Possible Mechanisms Underlying Bystander Effects}

The results of these experiments suggest that:

1. An irradiated cell can indeed send out a signal which can lead to an oncogenic response in a bystander cell, i.e., a cell whose nucleus is not hit.

2. In these experiments, the bystander effect is likely to be a binary "all or nothing" effect, in which a signal is sent out by cells traversed by single (or more) $\alpha$ particles, but more signal does not lead to any increase in oncogenic response.

3. The cell population may contain a small subpopulation that is hypersensitive to transformation by the bystander signal.

The binary nature of this bystander effect is suggested by the apparent saturation of the effect at low fluences (23). The rationale for the presence of a sensitive subpopulation that is highly responsive to bystander-induced oncogenesis is as follows: the phenomena of (1) an initial steep rise of response at low acute doses followed by a plateau and (2) an inverse dose-rate effect (increasing biological effect with increasing dose protraction) at low doses (e.g. ref. 24) are both characteristics that are consistent with the existence of a subpopulation of cells that is, at least at the time of irradiation, hypersensitive or already partially damaged (23).

\section{Possible Models of Bystander and Direct Effects}

While the results of experiments such as those described here suggest that bystander effects for end points relevant to cancer can be important, it is also clear that there must also be a component of radiobiological damage which is "direct", in the sense that it involves damage in a cell by a radiation track which deposits energy directly in that cell nucleus $(25,26)$. In an earlier analysis of the data described above, we discussed an approach to radiation-induced biological response, termed $\mathcal{B} a \mathcal{D}$, which incorporates both bystander and direct damage (23).

The $\mathcal{B}$ a $\mathcal{D}$ approach assumes that the overall response comes from both a bystander response in unhit cells and a direct response in hit cells. For the bystander component, it was assumed, based on the considerations discussed earlier, that the oncogenic bystander phenomenon is a binary "all or nothing" response to the bystander signal in a small sensitive subpopulation of cells (at least for high-LET radiation). It was further suggested that cells from this sensitive subpopulation are also very sensitive to direct hits from $\alpha$ particles, generally resulting in a directly hit sensitive cell being inactivated (23).

The resulting $\mathcal{B}$ a $\mathcal{D}$ model was used to analyze measured yields of oncogenic transformation per surviving cell from the three bystander protocols $[100 \%$ of cells irradiated with exact numbers of $\alpha$ particles, $10 \%$ of cells irradiated with exact numbers of $\alpha$ particles, cells exposed to a Poissondistributed number of traversals (23)]. The model has one free parameter relating to the direct component (the slope of the assumed linear response to high-LET radiation), and one free parameter relating to the bystander component (the proportion of cells that are highly sensitive to the bystander signal). A reasonable fit of the model to the data was seen, including the increased effect for a mean of one $\alpha$ particle compared to exactly one $\alpha$ particle, as well as the reversal of this difference with increasing numbers of $\alpha$ particles. Within the current approach, the reason for the difference in the observed transformation rate in the one case for $\alpha$ particles is the presence in the case of broad-beam (Poisson) irradiation of a significant proportion of cells which are not directly hit, a proportion which becomes very small with increasing fluence. Some of these unhit cells are assumed to be hypersensitive to the bystander signal. By contrast, when all the cell nuclei are hit directly, those cells that are hypersensitive to the bystander signal are largely inactivated.

With the two parameters of the model fixed by the fits to the data (21) for exact compared to Poisson-distributed numbers of particle traversals, the model was used to make predictions for the microbeam experiment (17), in which $10 \%$ of the cells are irradiated with exact numbers of $\alpha$ particles. The predictions of the model reproduce the trend of the data (23); in particular, the predictions are consistent with the prima facie anomalous observation that irradiation of $10 \%$ of cells with exactly one $\alpha$ particle apparently results in a bigger effect than does irradiation of $100 \%$ of cells with exactly one $\alpha$ particle. Analogous to the case of broad-beam irradiation, the putative reason for the different response is assumed to be the presence, when only 1 in 10 cells are directly irradiated, of nonhit hypersensitive cells that can contribute bystander effects. 


\section{DISCUSSION}

\section{Summary}

Summarized here is a quantitative model, based on the $\mathcal{B} \mathrm{D} \mathcal{D}$ approach (23), that incorporates radiobiological damage both from a bystander response to signals emitted by irradiated cells, and also from the direct traversal of ionizing radiations through cell nuclei. In the current model, no detailed signaling mechanisms were hypothesized, so the approach could, in principle, apply to the situation in which cells are in direct contact with one other, as well as to the situation in which the cells are further apart. In essence, it is assumed, at least for high-LET radiation, that this bystander effect is a binary "all or nothing" response to the bystander signal in a small sensitive subpopulation of cells. It is further assumed that cells from this bystandersignal-sensitive subpopulation are also very sensitive to direct hits from $\alpha$ particles, generally resulting in directly hit, bystander-signal-sensitive cells being inactivated.

The model was applied to a series of experiments on $\alpha$ particle-induced in vitro oncogenic transformation (using the immortalized mouse $\mathrm{C} 3 \mathrm{H} 10 \mathrm{~T}^{1} / 2$ cell line) with a singlecell/single-particle microbeam, as well as with broad-beam irradiation. The model was able to reproduce the main features of the data for both single and larger numbers of $\alpha$ particles.

\section{Relevance and Utility of Transformation Experiments with Immortalized Cells}

It is, of course, pertinent to consider whether oncogenic transformation in an immortalized mouse cell line is or could be useful in elucidating dose- or dose-rate-related trends of radiation-induced cancer in humans. Radiation carcinogenesis in vivo requires multiple genetic changes, which may be why transforming normal human cells in culture with radiation is difficult: The probabilities are just too small. The rationale underlying the use of immortalized cells in which some of these genetic events have already occurred is to separate out the radiation-dependent and the radiation-independent components of the overall process of radiation oncogenesis. This permits the study, with measurable frequencies, of the specifically radiation-related trends, such as dose-effect relationships, or dose-rate effects.

Of course, a price has to be paid with this approach, in that absolute risks cannot be assessed, since only a part of the entire process of radiation oncogenesis is being examined. Nevertheless, this approach has proven useful in the past for elucidating trends that are relevant to radiation carcinogenesis in humans. One example is the inverse doserate effect for radon: The dose/dose-rate trends for this phenomenon were quantified using experiments (24) with the immortalized $\mathrm{C} 3 \mathrm{H} 10 \mathrm{~T}^{1} / 2$ cell line, resulting in predictions as to the pattern of dose/time effects that would be expected in uranium miners (27). Subsequent epidemiological inves- tigations (28) showed that these dose/time patterns were indeed present in human populations.

\section{Constraints on Possible Models of Bystander Effects}

While some of the details of the model described here for this bystander response could change, some of its essential features currently appear to be constrained by the available data. Various other experiments on the bystander effect seem to suggest a rapid rise to a plateau at low doses, with little further dose dependence (see ref. 23). Sensitive subpopulations characteristically produce such plateaus in responses, although other phenomena, such as indirect, multistage pathways or adaptive responses, can also produce similar dose-effect relationships. The existence of an inverse dose-rate effect in other experiments (24) would also suggest a cell subpopulation which is hyper-responsive to a bystander signal. Typically, if a subpopulation has a saturated response for acute irradiation, but is restored by endogenous processes during prolonged irradiation, inverse dose-rate effects could result (29), which are indeed observed at low doses of high-LET radiation $(24,27,28)$.

More specifically, the results of the experiments with one $\alpha$ particle appear to constrain possible models significantly. Experiments in which all cells were traversed with one $\alpha$ particle appeared to produce lower risks than either those in which cells received a mean of one $\alpha$ particle or those where only 1 in 10 cells was traversed by one $\alpha$ particle. These results would suggest that when essentially all cells are directly struck by high-LET particles, bystander effects contribute little to the overall risk, but when significant numbers of cells are not directly hit, signals from hit cells to some of these untraversed cells can augment the overall risk significantly.

\section{Relevance to Risk Estimation for High-LET Low-Dose Radiation}

For exposure to high-LET particles, according to this picture, bystander effects are relevant primarily at low doses, i.e. where there are significant numbers of untraversed cells. Based on the fits to the data for in vitro oncogenic transformation, the bystander component appears to contribute only $6 \%$ of the measured transformation rate for a broadbeam irradiation with a mean of 4 particles [corresponding to a mean dose from $90 \mathrm{keV} / \mu \mathrm{m}$ particles of about $0.3 \mathrm{~Gy}$ (30)], increasing to $38 \%$ for a mean of 2 particles (mean dose $0.15 \mathrm{~Gy}$ ), and to $73 \%$ for a mean of 1 particle (mean dose 0.074 Gy).

If the trends postulated here were applicable in vivo, the consequences for risk estimation for low-dose radiation might be major, at least for high-LET radiations. The schematic in Fig. 4, based on the fits to the data for in vitro oncogenic transformation, suggests that a linear extrapolation of risks from data taken at radiobiologically and epidemiologically tractable intermediate doses of high-LET radiation (where the bystander effect may be negligible) to 


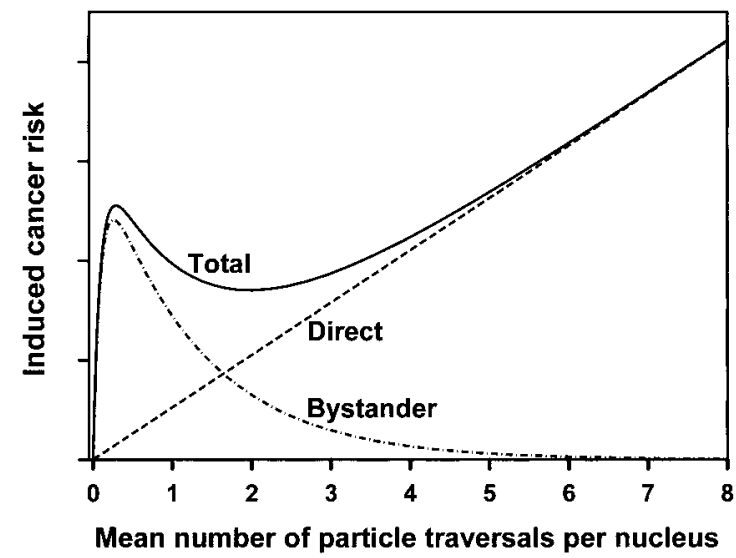

FIG. 4. Schematic of the possible relative contributions of the direct and bystander effects to the total cancer risk produced by radiation exposure on a Mars mission.

estimate risks at very low doses (where the bystander effect may be dominant) could underestimate the risk from low doses of high-LET radiation.

Our understanding of the bystander phenomenon is preliminary in nature, and the applicability of conclusions derived from in vitro studies to the situation in vivo is uncertain. However, these considerations would suggest caution in applying results derived from experiments with highLET radiation at doses above about $10 \mathrm{cGy}$ to risk estimation for a Mars mission or for domestic radon exposure.

It would be premature to consider rejecting current projections for the risk of exposure to high-LET, low-dose radiation solely on the basis of studies of the bystander phenomena for high-LET radiation in an in vitro rodent cell transformation system. The quantitative suggestions made here, however, are clearly testable.

\section{ACKNOWLEDGMENTS}

The analysis described here stems from experiments conceived and initiated by Eric Hall. In addition, this work would not have been possible without the experimental skills of Gerhard Randers-Pehrson and Satin Sawant, or without the insights of Rainer Sachs, Jack Little and Mike Cornforth. The Columbia University Radiological Research Accelerator Facility (RARAF) is an NIH Supported Resource Center. This work was supported by grants RR-11623, CA-49062, and CA-37967 from the National Institutes of Health, and by grant DE-FG02-98ER62686 from the U.S. Department of Energy.

Received: December 23, 2000; accepted: March 16, 2001

\section{REFERENCES}

1. G. D. Badhwar, Free space radiation environment. In Risk Evaluation of Cosmic-Ray Exposure in Long-term Manned Space Mission (K. Fujitaka, H. Majima, K. Ando, H. Yasuda and M. Suzuki, Eds.), pp. 17-31. Kodansha Scientific, Tokyo, 1999.

2. H. Nagasawa and J. B. Little, Induction of sister chromatid exchanges by extremely low doses of $\alpha$ particles. Cancer Res. 52, 6394-6396 (1992).

3. A. W. Hickman, R. J. Jaramillo, J. F. Lechner and N. F. Johnson,
Alpha-particle-induced p53 protein expression in a rat lung epithelial cell strain. Cancer Res. 54, 5797-5800 (1994).

4. A. Deshpande, E. H. Goodwin, S. M. Bailey, B. Marrone and B. Lehnert, Alpha-particle-induced sister chromatid exchange in normal human lung fibroblasts: Evidence for an extranuclear target. Radiat. Res. 145, 260-267 (1996).

5. B. E. Lehnert and E. H. Goodwin, A new mechanism for DNA alterations induced by alpha particles like those emitted by radon and radon progeny. Cancer Res. 57, 2164-2171 (1997).

6. C. B. Seymour and C. Mothersill, Delayed expression of lethal mutations and genomic instability in the progeny of human epithelial cells that survived in a bystander-killing environment. Radiat. Oncol. Invest. 5, 106-110 (1997).

7. C. Mothersill and C. B. Seymour, Cell-cell contact during gamma irradiation is not required to induce a bystander effect in normal human keratinocytes: Evidence for release during irradiation of a signal controlling survival into medium. Radiat. Res. 149, 256-262 (1998).

8. E. I. Azzam, S. M. de Toledo, T. Gooding and J. B. Little, Intercellular communication is involved in the bystander regulation of gene expression in human cells exposed to very low fluences of alpha particles. Radiat. Res. 150, 497-504 (1998).

9. K. M. Prise, O. V. Belyakov, M. Folkard and B. D. Michael, Studies of bystander effects in human fibroblasts using a charged particle microbeam. Int. J. Radiat. Biol. 74, 793-798 (1998).

10. S. A. Lorimore, M. A. Kadhim, D. A. Pocock, D. Papworth, D. L. Stevens, D. T. Goodhead and E. G. Wright, Chromosomal instability in the descendants of unirradiated surviving cells after $\alpha$-particle irradiation. Proc. Natl. Acad. Sci. USA 95, 5730-5733 (1998).

11. P. K. Narayanan, K. E. A. LaRue, E. H. Goodwin and B. E. Lehnert, Alpha particles initiate biological production of interleukin-8 in human cells. Radiat. Res. 152, 57-63 (1999).

12. H. Nagasawa and J. B. Little, Unexpected sensitivity to the induction of mutations by very low doses of alpha-particle radiation: Evidence for a bystander effect. Radiat. Res. 152, 552-557 (1999).

13. H. Zhou, G. Randers-Pehrson, C. A. Waldren, D. Vannais, E. J. Hall and T. K. Hei, Induction of a bystander mutagenic effect of alpha particles in mammalian cells. Proc. Natl. Acad. Sci. USA 97, 20992104 (2000).

14. P. K. Narayanan, E. H. Goodwin and B. E Lehnert, Alpha particles initiate biological production of superoxide anions and hydrogen peroxide in human cells. Cancer Res. 57, 3963-3971 (1997).

15. R. Iyer and B. E. Lehnert, Factors underlying the cell growth-related bystander responses to $\alpha$ particles. Cancer Res. 60, 1290-1298 (2000).

16. S. Sawant, G. Randers-Pehrson, C. R. Geard, D. J. Brenner and E. J. Hall, The bystander effect in radiation oncogenesis: I. Transformation in $\mathrm{C} 3 \mathrm{H} 10 \mathrm{~T}^{1 / 2}$ cells in vitro can be initiated in the unirradiated neighbors of irradiated cells. Radiat. Res. 155, 397-401 (2001).

17. S. Sawant, G. Randers-Pehrson, N. F. Metting, and E. J. Hall, Adaptive response and the bystander effect in $\mathrm{C} 3 \mathrm{H}_{10 \mathrm{~T}} 1 / 2$ cells in culture. Radiat. Res. 156, 177-180 (2001).

18. E. J. Hall and R. C. Miller, The how and why of in vitro oncogenic transformation. Radiat. Res. 87, 208-223 (1981).

19. G. Randers-Pehrson, C. R. Geard, G. Johnson and D. J. Brenner, Technical characteristics of the Columbia University single-ion microbeam. Radiat. Res. 156, 210-214 (2001).

20. Proceedings of the 4th International Workshop: Microbeam Probes of Cellular Radiation Response. Radiat. Res. 153, 220-238 (2000). [Extended abstracts]

21. R. C. Miller, G. Randers-Pehrson, C. R. Geard, E. J. Hall and D. J. Brenner, The oncogenic transforming potential of the passage of single alpha particles through mammalian cell nuclei. Proc. Natl. Acad. Sci. USA 96, 19-22 (1999).

22. M. Zelen, The analysis of several $2 \times 2$ contingency tables. Biometrika 58, 129-137 (1971).

23. D. J. Brenner, J. B. Little and R. K. Sachs, The bystander effect in 
radiation oncogenesis: II. A quantitative model. Radiat. Res. 155, 402-408 (2001).

24. R. C. Miller, D. J. Brenner, G. Randers-Pehrson, S. A. Marino and E. J. Hall, The effects of the temporal distribution of dose on oncogenic transformation by neutrons and charged particles of intermediate LET. Radiat. Res. 124 (Suppl.), S62-S68 (1990).

25. R. E. Zirkle and W. Bloom, Irradiation of parts of individual cells. Science 117, 487-493 (1953).

26. C. B. Seymour and C. Mothersill, Relative contribution of bystander and targeted cell killing to the low-dose region of the radiation doseresponse curve. Radiat. Res. 153, 508-511 (2000).

27. D. J. Brenner, The significance of dose rate in assessing the hazards of domestic radon exposure. Health Phys. 67, 76-79 (1994).
28. J. H. Lubin, J. D. Boice, C. Edling, R. W. Hornung, G. Howe, E. Kunz, R. A. Kusiak, H. I. Morrison, E. P. Radford and S. X. Yao, Radon-exposed underground miners and inverse dose-rate (protraction enhancement) effects. Health Phys. 69, 494-500 (1995).

29. D. J. Brenner and R. K. Sachs, Protraction effects in radiation studies: Basic biophysics. Radiat. Res. 154, 736-737 (2000).

30. C. R. Geard, G. Randers-Pehrson, T. K. Hei, G. J. Jenkins, R. C. Miller, L. J. Wu, D. J. Brenner and E. J. Hall, Microbeam mediated cellular effects: Single $\alpha$ particle induced chromosomal damage, cell cycle delay, mutation and oncogenic transformation. In Microdosimetry, an Interdisciplinary Approach (D. T. Goodhead, P. O'Neill and H. G. Menzel, Eds.), pp. 327-330. Royal Society of Chemistry, Cambridge, 1997. 